\title{
Comparing the burdens of opportunistic infections among patients with systemic rheumatic diseases: a nationally representative cohort study
}

\author{
Chung-Yuan Hsu' ${ }^{1}$, Chi-Hua Ko ${ }^{2,3}$, Jiun-Ling Wang ${ }^{4}$, Tsai-Ching Hsu ${ }^{5}$ and Chun-Yu Lin ${ }^{4 *}$ (D)
}

\begin{abstract}
Objective: To estimate and compare the burdens of opportunistic infections and herpes zoster in real-world practice among patients with various systemic rheumatic diseases.

Methods: This 13-year cohort study used national health insurance data to compare the incidence rates (IRs) of nine opportunistic infections among patients with five rheumatic diseases. The analyses were stratified according to followup duration using Poisson regression, and Cox models were used to compare the risk of first opportunistic infection.

Results: During 2000-2013, we identified 76,966 patients who had polymyositis/dermatomyositis (PM/DM, 2270 cases), systemic lupus erythematosus (SLE, 15,961 cases), systemic sclerosis (SSc, 2071 cases), rheumatoid arthritis (RA, 38,355 cases), or primary Sjögren's syndrome (pSS, 18,309 cases). The IR of opportunistic infections was highest for PM/DM cases (61.3/1000 person-years, 95\% confidence interval [CI] 56.6-66.2), followed by SLE cases (43.1/1000 person-years, 95\% Cl 41.7-44.5), SSc cases (31.6/1000 person-years, 95\% Cl 28.3-35.1), RA cases (25.0/1000 person-years, 95\% Cl 24.425.7), and pSS cases (24.1/1000 person-years, 95\% Cl 23.1-25.2). Multivariable Cox analysis revealed that, relative to SLE, PM/DM was associated with a significantly higher risk of opportunistic infections (hazard ratio 1.18, 95\% Cl 1.08-1.29). The risk of opportunistic infections was highest during the first year after the diagnosis of all five rheumatic diseases.

Conclusions: The risk of opportunistic infection was highest for PM/DM, followed by SLE, SSc, RA, and pSS. Careful observation and preventive therapy for opportunistic infections may be warranted in selected PM/DM patients, especially during the first year after the diagnosis.
\end{abstract}

Keywords: Polymyositis, Dermatomyositis, Systemic lupus erythematosus, Systemic rheumatic disease, Opportunistic infection

\section{Introduction}

Infection remains a leading cause of morbidity, hospitalization, and mortality among patients with autoimmune rheumatic disease [1, 2]. Furthermore, the estimated rates of infectious complications can be $26-50 \%$ among patients with polymyositis/dermatomyositis (PM/ DM) or systemic lupus erythematosus (SLE) [3-5]. Several factors may influence the vulnerability of patients with

\footnotetext{
* Correspondence: linchunyumed@gmail.com

${ }^{4}$ Department of Internal Medicine, National Cheng Kung University Hospital, College of Medicine, National Cheng Kung University, No.138, Sheng Li Road, 704 Tainan, Taiwan

Full list of author information is available at the end of the article
}

rheumatic diseases, with infectious diseases being strongly associated with their frequent use of corticosteroids and immunosuppressive agents. There is also evidence that innate and adaptive immunity against various pathogens is impaired in patients with SLE [6]. Moreover, the development of infection in patients with rheumatic diseases leads to a much poorer prognosis relative to that of patients without infectious diseases $[2,7]$.

In addition to common infections, opportunistic infection (OI) has emerged as an important complication in developed countries [8]. Interestingly, the risks of herpes zoster and Pneumocystis jiroveci pneumonia are elevated among SLE patients $[9,10]$, while DM was recently shown to be

(c) The Author(s). 2019 Open Access This article is distributed under the terms of the Creative Commons Attribution 4.0 International License (http://creativecommons.org/licenses/by/4.0/), which permits unrestricted use, distribution, and 
associated with elevated rates of herpes zoster and tuberculosis [11, 12]. However, most previous studies regarding the relationship between rheumatic diseases and OI were focused on SLE and were limited by their small-scale or single-centre designs. Furthermore, there is scarce research regarding the incidence rates (IRs) of OI in other major connective tissue diseases, such as systemic sclerosis (SSc) and primary Sjögren's syndrome (pSS). Moreover, among patients with PM/DM or SLE, there are no large-scale studies regarding the incidences of other OIs (e.g. aspergillosis, cryptococcosis, non-tuberculous mycobacteria, and cytomegalovirus infection). We are also unaware of any studies regarding whether the burden of OI varies among different connective tissue diseases. Therefore, the present study aimed to determine the incidence rates of various OIs in real-world practice among Taiwanese patients with five systemic rheumatic diseases (SLE, PM/DM, SSc, pSS, and rheumatoid arthritis [RA]). We also compared the risks of OIs between these rheumatic diseases.

\section{Methods \\ Data source}

This retrospective cohort study evaluated data from Taiwan's National Health Insurance Research Database (NHIRD; http://nhird.nhri.org.tw/en/index.html), which is maintained by Taiwan's National Health Research Institutes (NHRI). The NHIRD contains detailed demographic and healthcare information, including relevant diagnostic and procedural codes, for $>23,000,000$ individuals (approximately $99 \%$ of Taiwan's population). Furthermore, the size of the NHIRD has led to its extensive use for epidemiological studies [13], which have validated its accuracy for identifying major diseases, such as diabetes mellitus and cerebrovascular disease $[14,15]$. The study's retrospective protocol to evaluate de-identified secondary data was approved by the institutional review board of National Cheng Kung University Hospital (B-EX-108-012).

\section{Patients}

The five rheumatic diseases were identified using codes from the International Classification of Diseases, ninth revision, clinical modification (ICD-9-CM). Thus, inpatient and outpatient care claims were searched to identify cases involving SLE (710.0), RA (714.0), SSc (710.1), pSS (710.2), and PM/DM (710.4 and 710.3). To increase the specificity of case ascertainment for the five rheumatic diseases, we also evaluated catastrophic illness certificates, which are issued by the Bureau of National Health Insurance to patients with autoimmune diseases (e.g. SLE, RA, PM/DM, $\mathrm{pSS}$, or SSc) in order to exempt them from co-payment requirements for related medical care. The certificate is only issued to the patient when their medical records, laboratory data, and imaging results have been reviewed by two independent rheumatologists, who confirm that the corresponding classification criteria have been fulfilled. For example, the certificate can be issued to SLE patients when their symptoms, laboratory findings, and radiographic findings fulfill the 1997 American College of Rheumatology Revised Criteria for Classification of Systemic Lupus Erythematosus [16]. In RA cases, the American Rheumatism Association 1987 revised criteria or the 2010 American College of Rheumatology/European League Against Rheumatism criteria must be fulfilled [17, 18]. The revised American-European Consensus Group Classification Criteria or the European classification criteria are used for pSS $[19,20]$, the 1980 systemic sclerosis classification criteria are used for SSc [21], and Bohan and Peter's criteria are used for PM/DM [22, 23]. Patients without catastrophic illness certificates were excluded from our study.

The present study only included incident cases of autoimmune rheumatic diseases and excluded patients with $\geq 2$ rheumatic diagnoses to ensure that we did not consider patients with secondary Sjögren's syndrome or overlapping syndromes. The index date was defined as the first diagnosis of autoimmune disease between January 1, 2000, and December 31, 2013. Patients were followed up until the last episode of OI, death, or the end date (December 31, 2013).

\section{Identification of OI}

Based on the ICD-9-CM codes and the 2015 consensus recommendations for infection reporting [8], the OI types were defined as candidiasis (112), aspergillosis (117.3, 484.6), Cryptococcus infection $(117.5,321.0)$, Pneumocystis jiroveci pneumonia (136.3), cytomegalovirus infection (078.5, 771.1), salmonellosis (003), tuberculosis (010-018), non-tuberculous Mycobacterium infection (031.0, 031.2, 031.8, 031.9), herpes zoster (053), toxoplasmosis (130), coccidioidomycosis (114), and histoplasmosis (115). These codes for OI were generally considered to be accurate in the administrative database $[24,25]$, although case ascertainment was improved by restricting the eligible cases to those that involved inpatient claims using these codes, with the exception of herpes zoster, which generally does not require hospitalization. Patients were allowed to have multiple OI types or multiple episodes of the same OI, although only the first episode for each OI type was considered in the analysis of cases with multiple episodes of the same OI. Patients who had experienced OI before the index date were excluded from the analysis.

\section{Covariate information}

Patient characteristics (age, sex, income level, and comorbidities) were retrieved, and income level was used as a surrogate for socioeconomic status by categorizing the average monthly income as low $(\leq 19,200$ New Taiwan dollars [NTDs]), intermediate (19,201-40,000 NTD), and high (> 40,000 NTD). The selected baseline comorbidities were 
identified using ICD-9-CM codes for diabetes (250), chronic kidney disease (580-587), hypertension (401-405), ischaemic heart disease (410-414), cancer (140-208), dyslipidaemia (272), congestive heart failure (428), chronic obstructive lung disease (491, 492, 496), cerebrovascular disease (430-438), peripheral artery disease (443), liver cirrhosis $(571.2,571.5,571.6)$, hepatitis $\mathrm{B}$ virus infection (070.2, 070.3, v02.61), hepatitis C virus infection (070.41, $070.44,070.51,070.54,070.70,070.71, \mathrm{v02.62)}$, dementia (290, 294.1, 294.2, 331.0), and depression (296.2, 296.3, 311 . Comorbidities were considered present if the corresponding code was used for a single inpatient claim or $\geq 3$ outpatient visits. We also recorded the use of various medications within 90 days of the index date, including systemic corticosteroid, cyclophosphamide, methotrexate, azathioprine, cyclosporine, and leflunomide.

\section{Statistical analysis}

Demographic data and baseline comorbidities were presented as mean \pm standard deviation for continuous variables or as number (percentage) for categorical variables. These variables were then analysed using Student's independent $t$ test or Pearson's chi-squared test. Incidence rates (IRs) for overall OIs, and individual OI types, were estimated by dividing the total number of OI episodes by the relevant person-years value during the observation period. The time from rheumatic disease diagnosis to OI occurrence was stratified as $0-1$ year, $1-2$ years, 2-3 years, 4-5 years, and $>5$ years, and the incidence rate ratios (IRRs) for OI at the various follow-up times were estimated using Poisson regression. The Kaplan-Meier method and logrank test were used to identify differences in the cumulative incidences of OI between the five rheumatic diseases. Cox proportional hazard regression analyses were performed to estimate the effects of each disease on the risk of the first OI episode, after adjusting for age, sex, income level, and comorbidities. Crude and adjusted hazard ratios (HRs and aHRs) with 95\% confidence intervals (CIs) were used to describe the magnitudes of these effects.

\section{Sensitivity analysis}

To investigate the robustness of the main findings, sensitivity analyses were designed by excluding herpes zoster from the definition of OI. The same methods were then used to determine estimates of OI risk among patients with the various rheumatic diseases. An additional sensitivity analysis was performed to generate subdistribution hazard ratios (sHRs) via the competing risk regression model using Fine and Gray's method [26], with death as the competing risk. A two-sided $P$ value of $<0.05$ was considered significant. All data management and statistical analyses were performed using Stata 13 software (StataCorp, College Station, TX, USA).

\section{Results}

\section{Patient characteristics}

Between January 1, 2000, and December 31, 2013, we identified 76,966 patients with rheumatic diseases, including 15 , 961 SLE cases, 38,355 RA cases, 18,309 pSS cases, 2071 SSc cases, and 2270 PM/DM cases. Table 1 shows the patients' demographic characteristics, comorbidities, medications, and mean follow-up duration. Female sex was the predominant factor for all five rheumatic diseases. The highest female-to-male ratios were observed for SLE and pSS. The mean age was lowest in SLE cases (37.2 \pm 17.0 years) and was highest in pSS cases (54.7 \pm 14.3 years). Diabetes, hypertension, and dyslipidaemia were the most common comorbidities, and the proportion of patients with cancer was highest among patients with PM/DM, while that of patients with depression was highest among patients with pSS. Patients with RA, SLE, and SSc had longer follow-up times than those with PM/DM and pSS. Additional file 1: Table S1 shows the calendar year distributions of the index dates for patients with each rheumatic disease.

\section{Incidence of $\mathrm{OI}$}

We identified 13,002 episodes in 11,554 patients with OI. Among these patients, 10,341 cases $(89.5 \%)$ involved 1 episode, 1020 cases (8.8\%) involved 2 episodes, and 193 cases $(1.7 \%)$ involved $\geq 3$ episodes. Table 2 shows the IR and 95\% CI values for total OI and the various OI types according to the patients' rheumatic diseases, although $<2$ events were detected for toxoplasmosis, coccidioidomycosis, and histoplasmosis, which were omitted from the analyses. The highest IR for total OI was observed in PM/DM cases (61.3/1000 person-years, 95\% CI 56.6-66.2), which were followed by SLE cases $(43.1 / 1000$ person-years, 95\% CI 41.7-44.5), SSc cases $(31.6 / 1000$ person-years, 95\% CI 28.3-35.1), RA cases (25.0/1000 person-years, 95\% CI 24.4-25.7), and pSS cases (24.1/ 1000 person-years, 95\% CI 23.1-25.2). Relative to the SLE cohort, the IRR for OI in the PM/DM cohort was 1.42 (95\% CI 1.31-1.55) (Table 3). Sub-analyses of the OI types revealed generally similar rankings from highest to lowest (PM/DM followed by SLE, SSc, RA, and pSS), with the exception that the IR for salmonellosis was highest in the SLE cohort.

Table 4 shows the IRRs for OI according to the follow-up period. The risk of OI was highest during the first year after the diagnosis of the rheumatic diseases, with gradually decreasing risk over time. The lowest IR values for OI were observed at $>5$ years after the diagnosis of PM/DM, SLE, RA, and pSS.

Cumulative incidence and relative hazard of the first Ol event Figure 1 shows the cumulative incidences of total OI over 10 years in the rheumatic disease cohorts. The highest risk was observed in the PM/DM cohort, which 
Table 1 Patient characteristics according to systemic rheumatic disease

\begin{tabular}{|c|c|c|c|c|c|}
\hline Variable & $\operatorname{SLE}(N=15,961)$ & PM/DM $(N=2270)$ & $\operatorname{SSC}(N=2071)$ & RA $(N=38,355)$ & $\operatorname{pSS}(N=18,309)$ \\
\hline \multicolumn{6}{|l|}{ Sex, $n(\%)$} \\
\hline Male & $2027(12.7)$ & $760(33.5)$ & $544(26.3)$ & $9013(23.5)$ & $2032(11.1)$ \\
\hline Female & $13,934(87.3)$ & $1510(66.5)$ & $1527(73.7)$ & $29,342(76.5)$ & $16,277(88.9)$ \\
\hline Age in years, mean $\pm S D$ & $37.2 \pm 17.0$ & $47.8 \pm 17.7$ & $51.8 \pm 15.3$ & $53.3 \pm 15.4$ & $54.7 \pm 14.3$ \\
\hline \multicolumn{6}{|l|}{ Age group, $n(\%)$} \\
\hline $0-16$ years & $1342(8.4)$ & $148(6.5)$ & $32(1.6)$ & $712(1.8)$ & $42(0.2)$ \\
\hline $16-45$ years & $9853(61.7)$ & 767 (33.8) & $610(29.4)$ & $9688(25.3)$ & $4363(23.8)$ \\
\hline $45-65$ years & $3512(22.1)$ & $975(43.0)$ & $1000(48.3)$ & $19,105(49.8)$ & $9456(51.6)$ \\
\hline$>65$ years & $1254(7.8)$ & $380(16.7)$ & $429(20.7)$ & $8850(23.1)$ & $4448(24.4)$ \\
\hline \multicolumn{6}{|l|}{ Income in NTD, $n$ (\%) } \\
\hline Low $(<19,200)$ & $8092(50.7)$ & $1270(56.0)$ & $1129(54.5)$ & 20,647 (53.8) & $9521(52.0)$ \\
\hline Intermediate $(19,201-40,000)$ & $6071(38.1)$ & 747 (32.9) & $721(34.8)$ & $14,024(36.5)$ & $6526(35.6)$ \\
\hline High $(>40,000)$ & $1798(11.2)$ & $253(11.1)$ & $221(10.7)$ & $3684(9.7)$ & $2262(12.4)$ \\
\hline \multicolumn{6}{|l|}{ Comorbidities, n (\%) } \\
\hline Diabetes mellitus & $807(5.1)$ & $255(11.3)$ & $226(11.0)$ & $4528(11.8)$ & $2116(11.5)$ \\
\hline Hypertension & $2574(16.1)$ & $536(23.6)$ & $556(26.8)$ & $10,485(27.3)$ & $5277(28.8)$ \\
\hline Chronic kidney disease & $1390(8.7)$ & $99(4.4)$ & $147(7.1)$ & $1839(4.8)$ & $1064(5.8)$ \\
\hline COPD & $862(5.4)$ & $240(10.6)$ & $273(13.2)$ & $3930(10.3)$ & $2218(12.1)$ \\
\hline Ischaemic heart disease & $971(6.1)$ & $264(11.6)$ & $282(13.6)$ & $4643(12.1)$ & $2892(15.9)$ \\
\hline Dyslipidaemia & $1202(7.5)$ & $381(16.8)$ & $315(15.3)$ & $5743(14.9)$ & 3634 (19.8) \\
\hline $\mathrm{CHF}$ & $541(3.4)$ & $105(4.6)$ & $147(7.1)$ & $1303(3.4)$ & $584(3.2)$ \\
\hline Liver cirrhosis & $270(1.69)$ & $37(1.6)$ & $58(2.8)$ & $444(1.1)$ & $401(2.2)$ \\
\hline Cerebrovascular disease & $7.3(4.4)$ & $101(4.5)$ & $126(6.1)$ & $2235(5.8)$ & $1658(9.1)$ \\
\hline Cancer & $739(4.6)$ & $279(12.3)$ & $128(6.2)$ & $2116(5.5)$ & $1567(8.5)$ \\
\hline Peripheral artery disease & $942(5.9)$ & $140(6.2)$ & $624(30.1)$ & $1314(3.4)$ & $1145(6.25)$ \\
\hline HBV infection & $317(2.0)$ & $113(4.9)$ & $46(2.2)$ & $1118(2.9)$ & 727 (3.9) \\
\hline HCV infection & 249 (1.6) & $54(2.4)$ & $39(1.9)$ & $877(2.3)$ & 705 (3.8) \\
\hline Depression & 735 (4.6) & $109(4.8)$ & $110(5.3)$ & $2265(5.9)$ & $2262(12.3)$ \\
\hline Dementia & $92(0.6)$ & $13(0.6)$ & $12(0.6)$ & $322(0.8)$ & $279(1.5)$ \\
\hline \multicolumn{6}{|l|}{ Medications, $n(\%)$} \\
\hline Corticosteroid & $13,846(86.7)$ & $2146(94.5)$ & $1442(69.6)$ & $29,284(76.3)$ & $8912(48.7)$ \\
\hline Cyclophosphamide & $1320(8.3)$ & $208(9.2)$ & $186(9.0)$ & $192(0.5)$ & $229(1.25)$ \\
\hline Methotrexate & $626(3.9)$ & $668(29.4)$ & $155(7.5)$ & $21,310(55.6)$ & $829(4.5)$ \\
\hline Azathioprine & 3965 (24.8) & $708(31.2)$ & $166(8.0)$ & $621(1.6)$ & $1104(6.0)$ \\
\hline Cyclosporine & $270(1.7)$ & $99(4.4)$ & $37(1.8)$ & $1105(2.9)$ & $104(0.57)$ \\
\hline Leflunomide & $35(0.21)$ & $15(0.7)$ & $11(0.5)$ & $1914(5.0)$ & $108(0.59)$ \\
\hline Length of follow-up (years), mean $\pm S D$ & $5.6 \pm 4.2$ & $4.5 \pm 4.1$ & $5.3 \pm 4.0$ & $5.9 \pm 3.9$ & $4.5 \pm 3.5$ \\
\hline
\end{tabular}

SLE systemic lupus erythematosus, PM/DM polymyositis/dermatomyositis, $R A$ rheumatoid arthritis, $p S S$ primary Sjögren's syndrome, SSC systemic sclerosis, $S D$ standard deviation, NTD New Taiwan dollars, COPD chronic obstructive pulmonary disease, CHF congestive heart failure, HBV hepatitis B virus, HCV hepatitis $C$ virus

was followed by the SLE cohort, the SSc cohort, the RA cohort, and the pSS cohort (log-rank $P<0.001)$. We also examined the cumulative incidences of non-herpes zoster OIs, fungal OIs, and non-Candida fungal infections, which revealed similar risk patterns (Fig. 1b-d).
The Cox regression model, which was adjusted for age, sex, income level, and comorbidities, revealed that the risk of the first OI event was 18\% higher in the PM/ DM group than in the SLE group (aHR 1.18, 95\% CI 1.08-1.29). Relative to the SLE group, reduced risks of 
Table 2 Incidence rates of opportunistic infections according to systemic rheumatic disease

\begin{tabular}{|c|c|c|c|c|c|c|c|c|c|c|}
\hline & \multicolumn{2}{|c|}{$\begin{array}{l}\text { SLE }(N=15,961 \\
P Y=89,256)\end{array}$} & \multicolumn{2}{|c|}{$\begin{array}{l}\text { PM/DM }(N=2270 \\
P Y=10,252)\end{array}$} & \multicolumn{2}{|c|}{$\begin{array}{l}\text { SSC }(N=2071 \\
\text { PY }=10,868)\end{array}$} & \multicolumn{2}{|c|}{$\begin{array}{l}\text { RA }(N=38,355 \\
P Y=22,7549)\end{array}$} & \multicolumn{2}{|c|}{$\begin{array}{l}\text { pSS }(N=18,309, \\
P Y=81,995)\end{array}$} \\
\hline & Events & IR (95\% Cl) & Events & IR (95\% Cl) & Events & IR (95\% Cl) & Events & IR (95\% CI) & Events & IR (95\% Cl) \\
\hline \multicolumn{11}{|l|}{ Fungus } \\
\hline Aspergillus & 16 & $0.18(0.10-0.29)$ & 6 & $0.59(0.22-1.27)$ & 1 & $0.09(0.01-0.51)$ & 22 & $0.10(0.06-0.15)$ & 5 & $0.06(0.02-0.14)$ \\
\hline Candidiasis & 388 & $4.35(3.93-4.80)$ & 85 & $8.29(6.62-10.25)$ & 49 & $4.51(3.34-5.96)$ & 426 & $1.87(1.70-2.06)$ & 145 & $1.77(1.49-2.08)$ \\
\hline Cryptococcus & 39 & $0.44(0.31-0.60)$ & 9 & $0.88(0.40-1.67)$ & 2 & $0.18(0.02-0.67)$ & 45 & $0.20(0.14-0.27)$ & 14 & $0.17(0.09-0.29)$ \\
\hline PJP & 45 & $0.51(0.37-0.67)$ & 18 & $1.76(1.04-2.78)$ & 4 & $0.37(0.10-0.94)$ & 18 & $0.08(0.05-0.13)$ & 9 & $0.11(0.05-0.21)$ \\
\hline Subtotal & 488 & $5.48(4.99-5.98)$ & 118 & $11.5(9.53-13.8)$ & 56 & $5.15(3.89-6.69)$ & 511 & $2.25(2.06-2.45)$ & 173 & $2.11(1.81-2.45)$ \\
\hline CMV & 100 & $1.12(0.91-1.37)$ & 18 & $1.76(1.04-2.78)$ & 4 & $0.37(0.10-0.94)$ & 29 & $0.13(0.09-0.18)$ & 15 & $0.18(0.10-0.30)$ \\
\hline Salmonellosis & 272 & $3.05(2.70-3.43)$ & 30 & $2.93(1.97-4.18)$ & 11 & $1.01(0.51-1.81)$ & 118 & $0.52(0.43-0.62)$ & 27 & $0.33(0.22-0.48)$ \\
\hline Herpes zoster & 2580 & $28.9(27.8-30.0)$ & 377 & $36.8(33.2-40.7)$ & 216 & $19.9(17.3-22.7)$ & 4084 & $17.9(17.4-18.5)$ & 1502 & $18.3(17.4-19.3)$ \\
\hline TB & 341 & $3.82(3.43-4.25)$ & 71 & $6.93(5.41-8.74)$ & 47 & $4.32(3.18-5.75)$ & 839 & $3.69(3.44-3.95)$ & 213 & $2.60(2.26-2.97)$ \\
\hline NTM & 63 & $0.71(0.54-0.90)$ & 14 & $1.37(0.75-2.29)$ & 9 & $0.82(0.38-1.57)$ & 121 & $0.53(0.44-0.64)$ & 48 & $0.59(0.43-0.78)$ \\
\hline $\begin{array}{l}\text { Non-herpes zoster } \\
\text { infection }\end{array}$ & 1264 & $14.2(13.4-15.0)$ & 251 & $24.5(21.6-27.7)$ & 127 & $11.7(9.74-13.9)$ & 1617 & $7.10(6.76-7.40)$ & 476 & $5.81(5.30-6.35)$ \\
\hline Total & 3844 & $43.1(41.7-44.5)$ & 628 & $61.3(56.6-66.2)$ & 343 & $31.6(28.3-35.1)$ & 5701 & $25.0(24.4-25.7)$ & 1978 & $24.1(23.1-25.2)$ \\
\hline
\end{tabular}

Incidence rates (IRs) are reported as the number of cases per 1000 person-years

SLE systemic lupus erythematosus, RA rheumatoid arthritis, $p S S$ primary Sjögren's syndrome, SSc systemic sclerosis, $P M / D M$ polymyositis/dermatomyositis, $P J P$ Pneumocystis jiroveci pneumonia, CMV cytomegalovirus, TB tuberculosis, NTM non-tuberculosis mycobacterium, $P Y$ person-years

OI were observed in the SSc group (aHR 0.58, 95\% CI 0.51-0.65), the RA group (aHR 0.46, 95\% CI 0.44-0.48), and the pSS group (aHR 0.42, 95\% CI 0.39-0.44). Similar results were observed in the sensitivity analyses when herpes zoster was excluded from the outcome measurement (Table 5). The sensitivity analysis using the multivariable competing risk regression model also revealed that the PM/DM cohort had a significantly higher risk of OI than the SLE cohort (sHR 1.17, 95\% CI 1.07-1.29), while lower risks of OI were observed in the SSc cohort

Table 3 Unadjusted incidence rate ratio for total and nonherpes zoster opportunistic infections in patients with different systemic rheumatic diseases

\begin{tabular}{llll}
\hline & Incidence rate ratio & $95 \% \mathrm{Cl}$ & $P$ value \\
\hline \multicolumn{2}{l}{ Total opportunistic infections } & & \\
SLE & 1 (reference) & - & - \\
PM/DM & 1.42 & $1.31-1.55$ & $<0.001$ \\
SSC & 0.73 & $0.65-0.85$ & $<0.001$ \\
RA & 0.58 & $0.56-0.61$ & $<0.001$ \\
PSS & 0.56 & $0.53-0.59$ & $<0.001$ \\
Non-herpes zoster opportunistic infections & & \\
SLE & 1 (reference) & - & - \\
PM/DM & 1.73 & $1.50-1.98$ & $<0.001$ \\
SSC & 0.83 & $0.68-0.99$ & 0.035 \\
RA & 0.50 & $0.46-0.54$ & $<0.001$ \\
pSS & 0.41 & $0.37-0.45$ & $<0.001$ \\
\hline
\end{tabular}

SLE systemic lupus erythematosus, $R A$ rheumatoid arthritis, $p S S$ primary Sjögren's syndrome, SSC systemic sclerosis, $P M / D M$ polymyositis/ dermatomyositis, $\mathrm{Cl}$ confidence interval
(sHR 0.67, 95\% CI 0.59-0.75), the RA cohort (sHR 0.59, 95\% CI 0.57-0.62), and the pSS cohort (sHR 0.56, 95\% CI 0.53-0.59).

\section{Discussion}

To the best of our knowledge, this is the first nationally representative study to investigate the incidence of various OI types, including invasive fungal infection, mycobacterium infection, salmonellosis, and cytomegalovirus infection, among patients with five major immunemediated diseases (PM/DM, SLE, RA, SSc, and pSS). Our study is also the first to demonstrate that the risks of OI vary for each specific disease, with the highest risk observed for PM/DM, followed by SLE, SSc, RA, and pSS. In addition, we found that the risk of OI was highest during the first year after the diagnosis of rheumatic disease, with the risk subsequently decreasing at longer intervals after the diagnosis.

Previous studies have indicated that SLE patients have higher rates of bacterial infection or OI than the general population [1,2], which has been attributed to various factors. For example, several immune abnormalities have been reported in SLE patients, including complement deficiency [27], complement receptor deficiency $[9,28]$, defective chemotaxis and phagocytosis [7], decreased production of interleukin- 8 by polymorphonuclear leukocytes [29], and impaired activity of T-helper cells against viral antigens [30]. Furthermore, disease-related factors can also increase the risk of OI in patients with SLE, with their lupus activity index independently predicting the risk of hospitalization for infectious disease 
Table 4 Incidence rates and ratios for total opportunistic infections and non-herpes zoster infections according to systemic rheumatic disease and follow-up duration

\begin{tabular}{|c|c|c|c|c|c|c|c|}
\hline \multirow{2}{*}{$\begin{array}{l}\text { Follow-up } \\
\text { years }\end{array}$} & \multirow[t]{2}{*}{ Person-year } & \multicolumn{3}{|c|}{ Total opportunistic infections } & \multicolumn{3}{|c|}{ Non-herpes zoster opportunistic infections } \\
\hline & & Events & IR & IRR & Events & IR & IRR \\
\hline \multicolumn{8}{|l|}{ SLE } \\
\hline $0-1$ & 14,355 & 1212 & 84.4 & $2.76(2.53-2.99)$ & 425 & 29.6 & $2.86(2.48-3.31)$ \\
\hline $1-2$ & 12,495 & 540 & 43.2 & $1.41(1.27-1.57)$ & 168 & 13.5 & $1.30(1.07-1.58)$ \\
\hline $2-3$ & 11,050 & 418 & 37.8 & $1.24(1.10-1.38)$ & 126 & 11.4 & $1.10(0.89-1.36)$ \\
\hline $3-4$ & 9729 & 359 & 36.9 & $1.20(1.06-1.36)$ & 106 & 10.9 & $1.05(0.84-1.31)$ \\
\hline $4-5$ & 8483 & 300 & 35.3 & $1.15(1.01-1.31)$ & 96 & 11.3 & $1.09(0.86-1.38)$ \\
\hline$>5$ & 33,144 & 1015 & 30.6 & 1 (reference) & 343 & 10.4 & 1 (reference) \\
\hline Total & 89,256 & 3844 & 43.1 & - & 1264 & 14.2 & - \\
\hline \multicolumn{8}{|l|}{ RA } \\
\hline $0-1$ & 36,271 & 1123 & 30.9 & $1.32(1.23-1.43)$ & 331 & 9.13 & $1.44(1.25-1.66)$ \\
\hline $1-2$ & 32,300 & 825 & 25.5 & $1.09(1.01-1.18)$ & 226 & 7.00 & $1.10(0.94-1.29)$ \\
\hline $2-3$ & 28,646 & 676 & 23.6 & $1.01(0.92-1.10)$ & 201 & 7.02 & $1.11(0.94-1.31)$ \\
\hline $3-4$ & 25,375 & 596 & 23.5 & $1.01(0.91-1.10)$ & 165 & 6.51 & $1.03(0.86-1.22)$ \\
\hline $4-5$ & 22,283 & 547 & 24.5 & $1.05(0.95-1.15)$ & 170 & 7.63 & $1.20(1.01-1.43)$ \\
\hline$>5$ & 82,674 & 1934 & 23.4 & 1 (reference) & 524 & 6.34 & 1 (reference) \\
\hline Total & 227,549 & 5701 & 25.0 & - & 1617 & 7.10 & - \\
\hline \multicolumn{8}{|l|}{ pSS } \\
\hline $0-1$ & 16,658 & 528 & 31.7 & $1.54(1.36-1.75)$ & 152 & 9.12 & $1.85(1.44-2.37)$ \\
\hline $1-2$ & 13,798 & 355 & 25.7 & $1.25(1.08-1.43)$ & 71 & 5.15 & $1.04(0.76-1.41)$ \\
\hline $2-3$ & 11,438 & 242 & 21.1 & $1.03(0.88-1.20)$ & 49 & 4.28 & $0.87(0.61-1.22)$ \\
\hline $3-4$ & 9386 & 196 & 20.8 & $1.01(0.85-1.20)$ & 47 & 5.01 & $1.01(0.71-1.43)$ \\
\hline $4-5$ & 7657 & 182 & 23.7 & $1.15(0.97-1.37)$ & 43 & 5.62 & $1.14(0.78-1.62)$ \\
\hline$>5$ & 23,058 & 475 & 20.6 & 1 (reference) & 114 & 4.94 & 1 (reference) \\
\hline Total & 81,995 & 1978 & 24.1 & - & 476 & 5.81 & - \\
\hline \multicolumn{8}{|l|}{ SSC } \\
\hline $0-1$ & 1875 & 80 & 42.6 & $1.61(1.18-2.18)$ & 34 & 18.1 & $1.68(1.04-2.72)$ \\
\hline $1-2$ & 1600 & 57 & 35.6 & $1.34(0.95-1.88)$ & 18 & 11.2 & $1.04(0.56-1.86)$ \\
\hline $2-3$ & 1380 & 46 & 33.3 & $1.26(0.87-1.80)$ & 16 & 11.5 & $1.08(0.56-1.96)$ \\
\hline $3-4$ & 1183 & 40 & 33.8 & $1.27(0.86-1.85)$ & 11 & 9.29 & $0.86(0.40-1.71)$ \\
\hline $4-5$ & 1024 & 19 & 18.5 & $0.70(0.40-1.15)$ & 7 & 6.83 & $0.63(0.24-1.43)$ \\
\hline$>5$ & 3806 & 101 & 26.5 & 1 (reference) & 41 & 10.7 & 1 (reference) \\
\hline Total & 10,868 & 343 & 31.6 & - & 127 & 11.7 & - \\
\hline \multicolumn{8}{|l|}{ PM/DM } \\
\hline $0-1$ & 1882 & 277 & 147.2 & $5.47(4.31-6.98)$ & 137 & 72.8 & $11.2(7.14-18.2)$ \\
\hline $1-2$ & 1510 & 100 & 66.2 & $2.46(1.84-3.29)$ & 36 & 23.8 & $3.66(2.11-6.46)$ \\
\hline $2-3$ & 1277 & 78 & 61.1 & $2.27(1.66-3.09)$ & 23 & 18.8 & $2.76(1.48-5.15)$ \\
\hline $3-4$ & 1098 & 42 & 38.2 & $1.42(0.96-2.06)$ & 17 & 15.4 & $2.37(1.19-4.64)$ \\
\hline $4-5$ & 957 & 36 & 37.6 & $1.40(0.92-2.07)$ & 15 & 15.6 & $2.40(1.17-4.81)$ \\
\hline$>5$ & 3528 & 95 & 26.9 & 1 (reference) & 23 & 6.51 & 1 (reference) \\
\hline Total & 10,252 & 628 & 61.3 & - & 127 & 24.5 & - \\
\hline
\end{tabular}

IRs are reported as the number of cases per 1000 person-years

IR incidence rate, IRR incidence rate ratio, SLE systemic lupus erythematosus, RA rheumatoid arthritis, pSS primary Sjögren's syndrome, SSC systemic sclerosis, $P M / D M$ polymyositis/dermatomyositis 

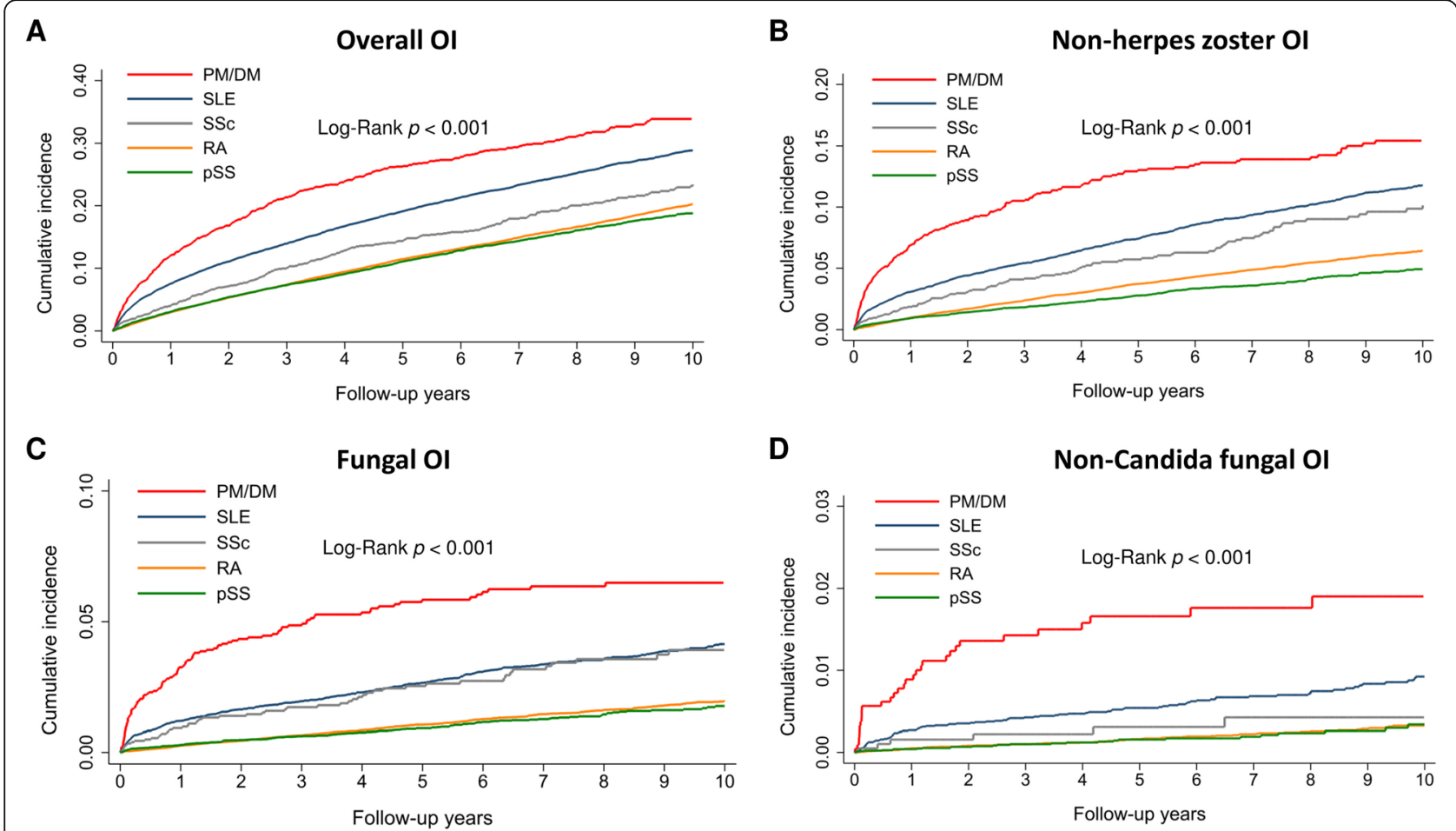

Fig. 1 Comparing the cumulative incidences of a overall opportunistic infections (OI), b non-herpes zoster Ols, c fungal Ols, and $\mathbf{d}$ non-Candida fungal Ols. The patients were grouped according to their systemic rheumatic diseases: polymyositis/dermatomyositis (PM/DM), systemic lupus erythematosus (SLE), systemic sclerosis (SSc), rheumatoid arthritis (RA), and primary Sjögren's syndrome (pSS)

[9]. Frequent use of glucocorticoids and immunosuppressive agents is also an important risk factor for unusual infection, and it has been reported that cyclophosphamide use for serious SLE manifestations is linked to fatal OIs [31]. A recent observational study also revealed that corticosteroid use had a dose-dependent effect on the rate of OIs [10].

One of the present study's main findings was that the IR of OI was significantly higher for PM/DM than for SLE, even after adjusting for age, sex, and comorbidities.

Table 5 Comparing the risks of opportunistic infection according to systemic rheumatic disease by Cox regression and competing risk regression models

\begin{tabular}{|c|c|c|c|c|}
\hline & \multicolumn{2}{|l|}{ Cox regression } & \multicolumn{2}{|c|}{ Competing risk regression } \\
\hline & Crude HR & Adjusted HR & Crude sHR & Adjusted sHR \\
\hline \multicolumn{5}{|c|}{ Opportunistic infections } \\
\hline SLE & 1 (reference) & 1 (reference) & 1 (reference) & 1 (reference) \\
\hline PM/DM & $1.38(1.26-1.51)$ & $1.18(1.08-1.29)$ & $1.22(1.11-1.34)$ & $1.17(1.07-1.29)$ \\
\hline SSC & $0.75(0.67-0.85)$ & $0.58(0.51-0.65)$ & $0.70(0.62-0.78)$ & $0.67(0.59-0.75)$ \\
\hline RA & $0.61(1.26-1.51)$ & $0.46(0.44-0.48)$ & $0.62(0.59-0.65)$ & $0.59(0.57-0.62)$ \\
\hline pSS & $0.57(0.54-0.60)$ & $0.42(0.39-0.44)$ & $0.58(0.55-0.61)$ & $0.56(0.53-0.59)$ \\
\hline \multicolumn{5}{|c|}{ Non-herpes zoster opportunistic infections } \\
\hline SLE & 1 (reference) & 1 (reference) & 1 (reference) & 1 (reference) \\
\hline PM/DM & $1.63(1.41-1.89)$ & $1.22(1.05-1.42)$ & $1.44(1.24-1.68)$ & $1.22(1.05-1.43)$ \\
\hline SSC & $0.83(0.68-1.01)$ & $0.55(0.45-0.67)$ & $0.77(0.64-0.93)$ & $0.65(0.54-0.79)$ \\
\hline RA & $0.50(0.47-0.54)$ & $0.33(0.30-0.36)$ & $0.51(0.47-0.55)$ & $0.45(0.42-0.49)$ \\
\hline pSS & $0.38(0.34-0.42)$ & $0.25(0.22-0.28)$ & $0.39(0.34-0.43)$ & $0.35(0.31-0.39)$ \\
\hline
\end{tabular}

HR hazard ratio, SHR subdistribution hazard ratio, SLE systemic lupus erythematosus, RA rheumatoid arthritis, pSS primary Sjögren's syndrome, SSC systemic sclerosis, $P M / D M$ polymyositis/dermatomyositis 
There are several possible explanations for this result. First, interstitial lung disease is a serious complication in up to $40-65 \%$ of $\mathrm{PM} / \mathrm{DM}$ cases [32, 33], and patients with interstitial lung disease may be vulnerable to pulmonary infections by Mycobacterium and Aspergillus species [34, 35]. Second, PM/DM patients often require more intensive immunosuppression than SLE patients, and fatal refractory interstitial lung disease associated with PM/DM is not uncommon, with Kameda et al. [36] reporting that treatment using cyclophosphamide plus glucocorticoids was only effective in $25 \%$ of these critical patients. Triple therapy using cyclophosphamide, cyclosporin A, and glucocorticoids has been suggested to increase the response rate in these refractory patients [37], although no combination treatments (e.g. $>2$ immunosuppressive agents) have been suggested for SLE patients, even in cases with lupus nephritis [38]. Thus, intensive immunosuppression may expose PM/DM patients to a significantly higher risk of OI than SLE patients. Third, PM/DM are strongly associated with a broad range of malignancies [39], which could contribute to the increased risk of OI through the use of cytotoxic anti-cancer therapies. Interestingly, malignancy can be present at the onset of idiopathic inflammatory myositis or may develop before or after the diagnosis of PM/ DM [39], although we found that the risk of OI remained higher for PM/DM patients than for SLE patients, even after adjusting our regression model for various cancer types. Fourth, involvement of the striated muscle at the oropharynx and upper third of the oesophagus can be observed in PM/DM patients, which can alter their ability to swallow and increase their risk of aspiration pneumonia [40]. Similarly, a small proportion of PM/DM patients experience thoracic muscle myopathy, which leads to ventilatory compromise, difficulty managing respiratory secretions, and an elevated risk of respiratory infection $[3,41]$.

Although infectious complications are more common in patients with connective tissue diseases, it is unclear whether the risk of OI varies over time. The present study revealed that the risk of OI was highest during the first year after the diagnosis of systemic rheumatic disease, especially among PM/DM patients, where the IR of OI during the first year after diagnosis was approximately 5.4 times greater than the IR at $>5$ years after diagnosis. Similarly, a French study of 156 PM/DM patients revealed that $62.5 \%$ of the OI events occurred during the first year after the PM/DM diagnosis [42]. Another cohort study explored the risk of herpes virus infection in 134 DM patients and also indicated that the IR was highest during the first year after DM diagnosis [43]. Other research has evaluated the courses of adult and juvenile DM patients, and the results suggested that disease activity was highest during the 6-12 months after the DM diagnosis, with improvement apparently accompanied by corticosteroid treatment [44-46]. Moreover, the required dosage of corticosteroid immunosuppression for DM was lower after 12 months of use and remained relatively constant until 36 months of use [46]. However, some studies have indicated that $11-30 \%$ of PM/DM patients developed OI before starting immunosuppressive therapy [42, 47]. Therefore, both high-dose corticosteroid treatment and high disease activity may contribute to the enhanced risk of OI during the first year after PM/DM diagnosis.

The introduction of biological agents has been a major advance in the treatment of RA [48]. For example, tumour necrosis factor alpha (TNF- $\alpha$ ) inhibitors have potent immunosuppressive effect in this setting and can prevent radiographic progression or induce clinical remission in RA patients [49]. However, infectious complications are important concerns when patients are receiving anti-TNF therapies [50], and there is evidence that anti-TNF therapies are associated with increased risks of serious infections that may require hospitalization [51-53]. In this context, etanercept and adalimumab were the first biologic agents approved for the treatment of severe RA in Taiwan and were widely used after 2004. However, we did not perform separate analyses of the risks of OI before and after the era of biological therapy in Taiwan, and caution should be exercised when interpreting our data regarding the incidence of OI in RA cases. It is important to note that RA patients receiving anti-TNF therapy or other biological agents may experience a higher risk of OI, relative to their apparent risk based on our findings.

Taiwan is a country with an intermediate burden of tuberculosis, based on an estimated IR of 68 cases per 100, 000 population in 2011 [54]. Our study revealed that incidences of tuberculosis in all five rheumatic diseases were several times higher than that in the general population, with the highest risk observed in the PM/DM cohort. These findings agree with the results from previous record-linkage studies conducted in Western countries [55, 56].

The strength of the present study lies in the use of a nationally representative data source with long-term followup data, which allowed us to examine the risks of overall and specific OIs according to five systemic rheumatic diseases. Nevertheless, our findings must be interpreted in the light of several limitations. First, the dataset lacked information regarding the activity or severity of the rheumatic diseases. Second, we did not incorporate variables regarding the exposure of glucocorticoids and immunosuppressants into our regression model, which precluded an analysis of their influence on the risk of OI. However, given the time-varying nature of medication use, it would be more appropriate to adopt a case-control study design for assessing the impact of immunosuppressive drugs. 
Third, we only counted the first episode in cases with multiple episodes of the same OI, which suggests that our calculated IR values might be underestimated.

\section{Conclusion}

This nationally representative cohort study revealed that patients with PM/DM had the highest risk of OI, followed by SLE, SSc, RA, and pSS, in order of decreasing risk. Furthermore, the highest risk of OI was observed during the first year after the diagnosis of systemic rheumatic disease, especially in cases of PM/ DM. These findings highlight the importance of monitoring for OI development during the treatment of these autoimmune rheumatic diseases, especially for patients with PM/DM.

\section{Supplementary information}

Supplementary information accompanies this paper at https://doi.org/10. 1186/s13075-019-1997-5.

Additional file 1: Table S1. Case distributions according to the calendar year of the index date and the rheumatic diseases.

\section{Abbreviations}

CHF: Congestive heart failure; CMV: Cytomegalovirus; COPD: Chronic obstructive pulmonary disease; HBV: Hepatitis B virus; HCV: Hepatitis C virus; HR: Hazard ratios; ICD-9-CM: International Classification of Diseases, ninth revision, clinical modification; IR: Incidence rates; IRR: Incidence rate ratio; NHIRD: National Health Insurance Research Database; NHRI: National Health Research Institutes; NTD: New Taiwan dollars; NTM: Non-tuberculosis mycobacterium; Ol: Opportunistic infection; PJP: Pneumocystis jiroveci pneumonia; PM/DM: Polymyositis/dermatomyositis; pSS: Primary Sjögren's syndrome; PY: Person-years; RA: Rheumatoid arthritis; sHR: Subdistribution hazard ratio; SD: Standard deviation; SLE: Systemic lupus erythematosus; SSc: Systemic sclerosis; TB: Tuberculosis; TNF-a: Tumour necrosis factor alpha

\section{Acknowledgements}

The study is based in part on data from the National Health Insurance Research Database, which was provided by the Bureau of National Health Insurance, Department of Health, and managed by the National Health Research Institutes. The interpretation and conclusions contained herein do not represent those of the Bureau of National Health Insurance, Department of Health, or National Health Research Institutes.

\section{Authors' contributions}

$\mathrm{CYL}$ is the guarantor of the paper and takes responsibility for the integrity of the work as a whole, from its inception to the published article. CYL and $\mathrm{CYH}$ contributed to the study design. All authors contributed to the acquisition of data. CYL and CYH contributed to the analysis and interpretation of the data. CYL contributed to the statistical analysis. CYL contributed to the manuscript preparation. CYL critically reviewed and edited the manuscript. All authors read and approved the final manuscript.

\section{Funding}

This study was supported by grant NCKUH-10803032 from the National Cheng Kung University Hospital, Tainan, Taiwan.

\section{Availability of data and materials}

The datasets used and/or analysed during the current study are available from the corresponding author on reasonable request.

\section{Ethics approval and consent to participate}

The Institutional review board of National Cheng Kung University Hospital approved this study (B-EX-108-012). Informed consent was not required because the datasets were devoid of personally identifiable information.
Consent for publication

Not applicable.

\section{Competing interests}

The authors declare that they have no competing interests.

\section{Author details}

${ }^{1}$ Division of Rheumatology, Allergy, and Immunology, Department of Internal Medicine, Kaohsiung Chang Gung Memorial Hospital and Chang Gung University College of Medicine, Kaohsiung, Taiwan. ${ }^{2}$ Department of Life Sciences, National Chung Hsing University, Taichung, Taiwan. ${ }^{3}$ Department of Rheumatology, Allergy and Immunology, Chang Gung Memorial Hospital, Yunlin, Taiwan. ${ }^{4}$ Department of Internal Medicine, National Cheng Kung University Hospital, College of Medicine, National Cheng Kung University, No.138, Sheng Li Road, 704 Tainan, Taiwan. ${ }^{5}$ Institute of Biochemistry, Microbiology and Immunology, Chung Shan Medical University, Taichung, Taiwan.

Received: 5 April 2019 Accepted: 5 September 2019

Published online: 12 October 2019

\section{References}

1. Feldman $\mathrm{CH}$, Hiraki LT, Winkelmayer WC, et al. Serious infections among adult Medicaid beneficiaries with systemic lupus erythematosus and lupus nephritis. Arthritis Rheumatol. 2015;67:1577-85.

2. Peng JM, Du B, Wang Q, et al. Dermatomyositis and polymyositis in the intensive care unit: a single-center retrospective cohort study of 102 patients. PLoS One. 2016;11:e0154441.

3. Marie I, Hatron PY, Dominique S, et al. Polymyositis and dermatomyositis: short term and long term outcome, and predictive factors of prognosis. J Rheumatol. 2001;28:2230-7.

4. Dankó K, Ponyi A, Constantin T, et al. Long-term survival of patients with idiopathic inflammatory myopathies according to clinical features: a longitudinal study of 162 cases. Medicine. 2004;83:35-42.

5. Zandman-Goddard G, Shoenfeld Y. Infections and SLE. Autoimmunity. 2005; 38:473-85.

6. Danza A, Ruiz-Irastorza G. Infection risk in systemic lupus erythematosus patients: susceptibility factors and preventive strategies. Lupus. 2013;22: 1286-94.

7. Petri M. Infection in systemic lupus erythematosus. Rheum Dis Clin North Am. 1998;24:423-56.

8. Winthrop KL, Novosad SA, Baddley JW, et al. Opportunistic infections and biologic therapies in immune-mediated inflammatory diseases: consensus recommendations for infection reporting during clinical trials and postmarketing surveillance. Ann Rheum Dis. 2015;74:2107-16.

9. Petri M, Genovese M. Incidence of and risk factors for hospitalizations in systemic lupus erythematosus: a prospective study of the Hopkins lupus cohort. J Rheumatol. 1992;19:1559-65.

10. Yang SC, Lai YY, Huang MC, Tsai CS, Wang JL. Corticosteroid dose and the risk of opportunistic infection in a national systemic lupus erythematosus cohort. Lupus. 2018;27:1819-27.

11. Tsai SY, Lin CL, Wong YC, et al. Increased risk of herpes zoster following dermatomyositis and polymyositis: a nationwide population-based cohort study. Medicine. 2015;94:e1138.

12. Wu PH, Lin YT, Yang YH, Lin YC, Lin YC. The increased risk of active tuberculosis disease in patients with dermatomyositis - a nationwide retrospective cohort study. Sci Rep. 2015;5:16303.

13. Chang SL, Huang YL, Lee MC, et al. Association of varicose veins with incident venous thromboembolism and peripheral artery disease. JAMA. 2018;319:807-17.

14. Cheng CL, Kao YH, Lin SJ, Lee CH, Lai ML. Validation of the national health insurance research database with ischemic stroke cases in Taiwan. Pharmacoepidemiol Drug Saf. 2011;20:236-42.

15. Lin CC, Lai MS, Syu CY, Chang SC, Tseng FY. Accuracy of diabetes diagnosis in health insurance claims data in Taiwan. J Formos Med Assoc. 2005;104: 157-63.

16. Hochberg MC. Updating the American College of Rheumatology revised criteria for the classification of systemic lupus erythematosus. Arthritis Rheum. 1997:40:1725. 
17. Arnett FC, Edworthy SM, Bloch DA, et al. The American Rheumatism Association 1987 revised criteria for the classification of rheumatoid arthritis. Arthritis Rheum. 1988;31:315-24.

18. Aletaha D, Neogi T, Silman AJ, et al. 2010 Rheumatoid arthritis classification criteria: an American College of Rheumatology/European League Against Rheumatism collaborative initiative. Arthritis Rheum. 2010;62:2569-81.

19. Vitali C, Bombardieri S, Moutsopoulos HM, et al. Preliminary criteria for the classification of Sjögren's syndrome. Results of a prospective concerted action supported by the European Community. Arthritis Rheum. 1993;36: 340-7.

20. Vitali C, Bombardieri S, Jonsson R, et al. Classification criteria for Sjögren's syndrome: a revised version of the European criteria proposed by the American-European Consensus Group. Ann Rheum Dis. 2002;61:554-8.

21. Preliminary criteria for the classification of systemic sclerosis (scleroderma). Subcommittee for scleroderma criteria of the American Rheumatism Association Diagnostic and Therapeutic Criteria Committee. Arthritis Rheum. 1980;23:581-90.

22. Bohan A, Peter JB. Polymyositis and dermatomyositis (first of two parts). N Engl J Med. 1975;292:344-7.

23. Bohan A, Peter JB. Polymyositis and dermatomyositis (second of two parts). N Engl J Med. 1975:292:403-7.

24. Schneeweiss S, Robicsek A, Scranton R, Zuckerman D, Solomon DH. Veteran's affairs hospital discharge databases coded serious bacterial infections accurately. J Clin Epidemiol. 2007;60:397-409.

25. Grijalva CG, Chung CP, Stein CM, et al. Computerized definitions showed high positive predictive values for identifying hospitalizations for congestive heart failure and selected infections in Medicaid enrollees with rheumatoid arthritis. Pharmacoepidemiol Drug Saf. 2008;17:890-5.

26. Fine JP, Gray RJ. A proportional hazards model for the subdistribution of a competing risk. J Am Stat Assoc. 1999;94:496-509.

27. Ross SC, Densen P. Complement deficiency states and infection: epidemiology, pathogenesis and consequences of neisserial and other infections in an immune deficiency. Medicine. 1984;63:243-73.

28. Wilson JG, Ratnoff WD, Schur PH, Fearon DT. Decreased expression of the $\mathrm{C} 3 \mathrm{~b} / \mathrm{C} 4 \mathrm{~b}$ receptor (CR1) and the $\mathrm{C} 3 \mathrm{~d}$ receptor (CR2) on $\mathrm{B}$ lymphocytes and of CR1 on neutrophils of patients with systemic lupus erythematosus. Arthritis Rheum. 1986;29:739-47.

29. Hsieh SC, Tsai CY, Sun KH, et al. Decreased spontaneous and lipopolysaccharide stimulated production of interleukin 8 by polymorphonuclear neutrophils of patients with active systemic lupus erythematosus. Clin Exp Rheumatol. 1994;12:627-33.

30. Bermas BL, Petri M, Goldman D, et al. T helper cell dysfunction in systemic lupus erythematosus (SLE): relation to disease activity. J Clin Immunol. 1994; 14:169-77.

31. Pryor BD, Bologna SG, Kahl LE. Risk factors for serious infection during treatment with cyclophosphamide and high-dose corticosteroids for systemic lupus erythematosus. Arthritis Rheum. 1996;39:1475-82.

32. Fathi M, Dastmalchi M, Rasmussen E, Lundberg IE, Tornling G. Interstitial lung disease, a common manifestation of newly diagnosed polymyositis and dermatomyositis. Ann Rheum Dis. 2004;63:297-301.

33. Kang $E H$, Lee $E B$, Shin $K C$, et al. Interstitial lung disease in patients with polymyositis, dermatomyositis and amyopathic dermatomyositis. Rheumatology. 2005:44:1282-6.

34. Smith NL, Denning DW. Underlying conditions in chronic pulmonary aspergillosis including simple aspergilloma. Eur Respir J. 2011;37:865-72.

35. Park SW, Song JW, Shim TS, et al. Mycobacterial pulmonary infections in patients with idiopathic pulmonary fibrosis. J Korean Med Sci. 2012; 27:896-900

36. Kameda $\mathrm{H}$, Nagasawa $\mathrm{H}$, Ogawa $\mathrm{H}$, et al. Combination therapy with corticosteroids, cyclosporin A, and intravenous pulse cyclophosphamide for acute/subacute interstitial pneumonia in patients with dermatomyositis. J Rheumatol. 2005;32:1719-26.

37. Miyazaki E, Ando M, Muramatsu T, et al. Early assessment of rapidly progressive interstitial pneumonia associated with amyopathic dermatomyositis. Clin Rheumatol. 2007;26:436-9.

38. Hahn BH, McMahon MA, Wilkinson A, et al. American College of Rheumatology guidelines for screening, treatment, and management of lupus nephritis. Arthritis Care Res. 2012;64:797-808.

39. Sigurgeirsson B, Lindelöf B, Edhag O, Allander E. Risk of cancer in patients with dermatomyositis or polymyositis. A population-based study. N Engl J Med. 1992;326:363-7.
40. Mugii N, Hasegawa M, Matsushita T, et al. Oropharyngeal dysphagia in dermatomyositis: associations with clinical and laboratory features including autoantibodies. PLoS One. 2016;11:e0154746.

41. Wu Q, Wedderburn LR, McCann LJ. Juvenile dermatomyositis: Latest advances. Best Pract Res Clin Rheumatol. 2017;31:535-57.

42. Marie I, Hachulla E, Chérin P, et al. Opportunistic infections in polymyositis and dermatomyositis. Arthritis Rheum. 2005;53:155-65.

43. Fardet $\mathrm{L}$, Rybojad M, Gain M, et al. Incidence, risk factors, and severity of herpesvirus infections in a cohort of 121 patients with primary dermatomyositis and dermatomyositis associated with a malignant neoplasm. Arch Dermatol. 2009;145:889-93.

44. Ramanan AV, Campbell-Webster N, Ota S, et al. The effectiveness of treating juvenile dermatomyositis with methotrexate and aggressively tapered corticosteroids. Arthritis Rheum. 2005;52:3570-8.

45. Schmeling H, Stephens S, Goia C, et al. Nailfold capillary density is importantly associated over time with muscle and skin disease activity in juvenile dermatomyositis. Rheumatology. 2011;50:885-93.

46. Johnson NE, Arnold WD, Hebert D, et al. Disease course and therapeutic approach in dermatomyositis: a four-center retrospective study of 100 patients. Neuromuscul Disord. 2015;25:625-31.

47. Viguier M, Fouéré $S$, de la Salmonière $P$, et al. Peripheral blood lymphocyte subset counts in patients with dermatomyositis: clinical correlations and changes following therapy. Medicine. 2003;82:82-6.

48. Davignon JL, Rauwel B, Degboé $Y$, et al. Modulation of T-cell responses by anti-tumor necrosis factor treatments in rheumatoid arthritis: a review. Arthritis Res Ther. 2018;20:229.

49. Stamm TA, Machold KP, Aletaha D, et al. Induction of sustained remission in early inflammatory arthritis with the combination of infliximab plus methotrexate: the DINORA trial. Arthritis Res Ther. 2018;20:174.

50. Pawar A, Desai RJ, Solomon DH, et al. Risk of serious infections in tocilizumab versus other biologic drugs in patients with rheumatoid arthritis: a multidatabase cohort study. Ann Rheum Dis. 2019;78:456-64.

51. Singh JA, Cameron C, Noorbaloochi S, et al. Risk of serious infection in biological treatment of patients with rheumatoid arthritis: a systematic review and meta-analysis. Lancet. 2015;386:258-65.

52. Galloway JB, Hyrich KL, Mercer LK, et al. Anti-TNF therapy is associated with an increased risk of serious infections in patients with rheumatoid arthritis especially in the first 6 months of treatment: updated results from the British Society for Rheumatology Biologics Register with special emphasis on risks in the elderly. Rheumatology (Oxford). 2011;50:124-31.

53. Smitten AL, Choi HK, Hochberg MC, et al. The risk of hospitalized infection in patients with rheumatoid arthritis. J Rheumatol. 2008;35:387-93.

54. Taiwan Centers for Disease Control. Taiwan Tuberculosis Control Report. 2013. https://www.cdc.gov.tw/uploads/files/201407/103228a0-fadd-47b0b056-8dedda9fce1d.pdf

55. Ramagopalan SV, Goldacre R, Skingsley A, Conlon C, Goldacre MJ. Associations between selected immune-mediated diseases and tuberculosis: record-linkage studies. BMC Med. 2013;11:97.

56. Airio A, Kauppi M, Kautiainen $H$, Hakala M, Kinnula V. High association of mycobacterial infections with polymyositis in a non-endemic country for tuberculosis. Ann Rheum Dis. 2007;66:1404-5.

\section{Publisher's Note}

Springer Nature remains neutral with regard to jurisdictional claims in published maps and institutional affiliations.

Ready to submit your research? Choose BMC and benefit from:

- fast, convenient online submission

- thorough peer review by experienced researchers in your field

- rapid publication on acceptance

- support for research data, including large and complex data types

- gold Open Access which fosters wider collaboration and increased citations

- maximum visibility for your research: over $100 \mathrm{M}$ website views per year

At $\mathrm{BMC}$, research is always in progress.

Learn more biomedcentral.com/submissions 